\title{
Mesoscopic Luttinger Liquid Theory in an Aharonov-Bohm Ring
}

\author{
Mun Dae Kim ${ }^{1,2}$, Sam Young $\mathrm{Cho}^{1,2}$, Chul Koo Kim ${ }^{1,2}$, and Kyun Nahm ${ }^{3}$ \\ ${ }^{1}$ Institute of Physics and Applied Physics, Yonsei University, Seoul 120-749, Korea \\ ${ }^{2}$ Center for Strongly Correlated Materials Research, \\ Seoul National University, Seoul 151-742, Korea \\ ${ }^{3}$ Department of Physics, Yonsei University, Wonju 220-710, Korea
}

(Dated: October 27, 2018)

\begin{abstract}
A careful study on the mesoscopic persistent current in a Luttinger liquid ring is carried out. It is shown that discreteness plays an important role in calculating the persistent current caused by the magnetic flux. At zero temperature, the current is shown to be independent of the interaction even when $g=\left(g_{2}-g_{4}\right) / 2$ is not zero. The current becomes enhanced at finite temperatures with respect to the non-interacting case, when the parameter $g$ is positive.
\end{abstract}

With rapid development of nano-fabrication technology much attention has been paid to the mesoscopic system which exhibits strong quantum phase coherence. Many efforts have been devoted to incorporate the interaction effect between electrons because they are strongly correlated in low dimensions. The Luttinger liquid(LL) theory [1, 2, 3, 4, 旬 is a standard method to investigate 1D interacting electron systems. Therefore, it became a starting point to study the mesoscopic property such as the persistent current $(\mathrm{PC})[6$.

Here, we consider the zero mode current in a mesoscopic LL ring without the Aharonov-Bohm(AB) flux and extend it to the persistent current produced by the $\mathrm{AB}$ flux. In the continuum limit of LL theory, the current is calculated through the continuity equation. In this approach, the mean current carries a prefactor, $v_{J}$, which is renormalized by interactions [1]. However, for a mesoscopic ring, the momentum level is discrete and, thus, the continuum approach is not valid. The mean current must be obtained from the zero mode. We show that the zero mode current operator does not depend on the interaction parameter.

In this study, we consider the case when leftright symmetry is absent and show the continuum approximation $\sqrt{6}]$ is not appropriate for the $\mathrm{PC}$ in the Luttinger liquid model, where discreteness plays a crucial role. In the present calculation, we take discreteness into the calculation explicitly and show that the calculation yields new results that, at zero temperature, the expectation value of the $\mathrm{PC}$ does not depend on the LL interaction with $g \geq 0$. At finite temperatures the interaction enhances the amplitude of $\mathrm{PC}$ with respect to that of the non-interaction case.

We, first, study the current in a mesoscopic LL ring without $\mathrm{AB}$ flux. Here and after, we consider the spinless particles, because the spin degrees do not give any qualitatively different results for the amplitude of the current. The current can be defined through the continuity equation such that $\frac{d}{d t} \rho(x)=i[H, \rho(x)]=\nabla j(x)$, where $\rho(x)=\sum_{p} e^{i p x} \rho(p) \equiv-\frac{1}{\pi} \partial_{x} \varphi(x)$ is the bosonic density operator in the LL. Then $j(x)$ is represented by

$$
j(x)=i\left[H, \sum_{p} \frac{1}{i p} e^{i p x} \rho_{p}\right]+\tilde{c},
$$

where $\tilde{c}$ is an operator independent of $x$. The mean current operator, $I$, can be defined by $I \equiv \frac{1}{L} \int_{0}^{L} d x^{\prime} j\left(x^{\prime}\right)$. In the limit when the level spacing $\Delta p \rightarrow 0$, the mean current $I$ is given by $I=\lim _{p \rightarrow 0} \frac{1}{p L}\left[H, \rho_{p}\right]$, which results that $I=v_{J} \frac{J}{L}$ with the interaction dependent prefactor $v_{J}$. However, for a mesoscopic wire, we cannot assume the continuum limit and, thus, should sum over the discrete $p$ values,

$$
\begin{aligned}
I & =\frac{1}{L} \int_{0}^{L} d x^{\prime}\left\{i \sum_{p \neq 0} \frac{1}{i p} e^{i p x^{\prime}}\left[H, \rho_{p}\right]+i\left[H, \frac{N}{L} x^{\prime}\right]+\tilde{c}\right\} \\
& =\tilde{c}
\end{aligned}
$$

since $[H, N]=0$. Here, $I=\tilde{c}$ is the uniform zero mode current which can not be obtained through the continuity equation, but only through the definition in terms of total momentum of electrons.

When the total particle number $N_{o}$ is odd, there is no current excitation at the ground state. The number of particles at the right(left) branch, $N_{o+}\left(N_{o-}\right)$, becomes $N_{o+}=N_{o-}=\left(N_{o}-1\right) / 2$, where we subtracted 1 , the number of particle at the zero momentum state. For even $N_{o}, N_{o+}=\left(N_{o}-1+k_{J}\right) / 2$ and $N_{o-}=\left(N_{o}-1-k_{J}\right) / 2$. If the right(left) branch has one more particle at the ground state, $k_{J}=1(-1)$. The case when $N_{o}$ is odd, corresponds to $k_{J}=0$. At finite temperatures, current excitation number $N_{+}\left(N_{-}\right)$is added to $N_{o+}\left(N_{o-}\right)$. For the non-interacting case, the zero mode current, $I$, can be calculated in terms of total momentum, $-\frac{e}{L} \sum_{n=-N_{o-}}^{N_{o+}+N_{+}} \frac{2 \pi}{L} n$, and represented by the excitation numbers,

$$
I=-\frac{e}{L} \frac{N_{o}}{2} \frac{2 \pi}{L}\left(J+k_{J}\right)=-\frac{e v_{F}}{L}\left(J+k_{J}\right) .
$$

Here, $J \equiv N_{+}-N_{-}$is the current excitation number and $N \equiv N_{+}+N_{-}$the charge excitation number. For an interacting case, this range of summation remains correct, 
if internal interactions do not change the total momentum of the system. The zero mode of the Hamiltonian exactly satisfies this condition. The interaction effect is included in obtaining the expectation value, $\langle I\rangle$. However, the current operator should have the interaction independent fermi velocity, $v_{F}$, as a prefactor instead of $v_{J}$. Here we define the fermi velocity such that $v_{F} \equiv \frac{N_{o}}{2} \frac{2 \pi}{L}$, since this corresponds to the product of the particle number at one branch with the momentum discreteness.

In the LL theory, the non-zero and the zero mode in the Hamiltonian are decoupled and the zero mode is given by $\frac{\pi}{2 L}\left(v_{F}+\frac{g_{4}-g_{2}}{2 \pi}\right)\left(J+\frac{2 \phi}{\phi_{o}}\right)^{2}+\frac{\pi}{2 L}\left(v_{F}+\frac{g_{4}+g_{2}}{2 \pi}\right) N^{2}$. The contribution of the flux in the kinetic part comes from changes in the momenta of electrons in accordance to the twisted boundary condition and changes continuously. But, in the interaction part, excitation energy is determined by the current and charge excitation number which are integers. In the continuum field theory, the particle distribution is continuous and, thus, the interaction energy can also be continuous. However, since we are now studying the mesoscopic regime, where the particle discreteness is crucial. We show in the following that a small level shift without charge or current excitation does not cause any change in the interaction energy.

In order to account the level discreteness correctly, the twisted boundary condition should be implemented from the beginning of the bosonization process. Also, a careful analysis on the discreteness of the particle distribution is required, since the parity effect comes from the discreteness of the particle numbers. When the fermion field is expanded, $\psi_{r}(x) \equiv\left(\frac{2 \pi}{L}\right)^{\frac{1}{2}} \sum_{k=-\infty}^{\infty} e^{-i k x} c_{k r}$, the twisted boundary condition gives a condition that $k=$ $\frac{2 \pi}{L}\left(n+\phi / \phi_{o}\right)[10]$. Here, $n$ is an integer. With this modification on $\mathrm{k}$, one can proceed the bosonization process exactly same as when $\phi=0$. Considering the flux range, $-\frac{\pi}{L}<\frac{\phi}{\phi_{o}} \frac{2 \pi}{L}<\frac{\pi}{L}$, we note that positive magnetic flux causes an upward(downward) shift of the right(left) branch electrons. Now, the zero mode contribution to the kinetic energy excitation can be obtained from the prescription $H_{k}^{o}=\sum_{r} H_{k r}^{o}$ with $H_{k r}^{o}=\sum k: c_{k r}^{\dagger} c_{k r}$ :, where :: signifies the normal ordering.

To obtain the zero mode bosonic form in $H_{k}^{o}$, we consider the ladder operator which increases the number of electrons above the Fermi level, such that $U_{r}\left|N_{r}, N_{-r}\right\rangle=$ $\left|N_{r}+1, N_{-r}\right\rangle$. The ladder operator can be constructed as $\sum_{k} c_{k p}^{\dagger} \delta\left[r k-\left(k_{F}+\frac{2 \pi}{L}\left(N_{r}-\frac{1}{2}\right)\right)\right]$ 1, 2], where $N_{r}$ represents the $N_{r}$ th excitation state in the $\mathrm{r}$ branch. Here, the energies are measured from the reference level, $v_{F} \frac{\pi}{L}$. The twisted boundary condition shifts the momentum levels by $\frac{2 \phi}{\phi_{0}} \frac{\pi}{L}$ and the ladder operator creates particles at the shifted levels such that,

$$
U_{r}=\sum_{k} c_{k p}^{\dagger} \delta\left[r k-\left(k_{F}+\frac{2 \pi}{L}\left(N_{r}-\frac{1}{2}+r \frac{\phi}{\phi_{o}}\right)\right)\right] .
$$

The zero mode part of the kinetic energy excitation at each branch is given by

$$
H_{k r}^{o}=\frac{2 \pi v_{F}}{L} \sum_{n=1}^{N_{r}}\left(n-\frac{1}{2}+r \frac{\phi}{\phi_{o}}\right)=\frac{\pi v_{F}}{L} N_{r}^{2}+r \frac{2 \pi v_{F}}{L} \frac{\phi}{\phi_{o}} N_{r}
$$

For the even parity, application of the magnetic flux removes the degeneracy of the uppermost particle occupation. Since positive $\phi$ shifts the level to the right side, the uppermost particle takes a level at the left branch. Thus, the energy cost for the excitation in the left branch become increased by the level width, $v_{F} \frac{2 \pi}{L}$, for each excited particle. Considering this effect and Eq. (5), we obtain for the kinetic part

$$
H_{k}^{o}=\frac{\pi v_{F}}{2 L} N^{2}+\frac{\pi v_{F}}{2 L}\left(\left(J+k_{J}\right)^{2}+2\left(J+k_{J}\right) \frac{2 \phi}{\phi_{o}}\right),
$$

where $k_{J}=-1(+1)$ for positive(negative) $\phi$ for the even parity and 0 for the odd parity.

The zero mode in the interacting part, $H_{i n t}^{o}$, is directly deducible from the Hamiltonian. The wave vector shift due to the twisted boundary condition makes change in the bosonic density operator as $\rho(p)=\sum_{k}$ : $c_{k+\frac{\phi}{\phi_{o}}}^{\dagger} \frac{2 \pi}{L}+p c_{k+\frac{\phi}{\phi_{o}} \frac{2 \pi}{L}}:$. We, thus, can write $H_{i n t}^{o}$ for the $p=0$ mode from the Hamiltonian,

$$
\begin{aligned}
& \frac{g_{2}}{2 L} \sum_{r, k, k^{\prime}}: c_{r, k+\frac{\phi}{\phi_{o}} \frac{2 \pi}{L}}^{\dagger} c_{r, k+\frac{\phi}{\phi_{o}} \frac{2 \pi}{L}}:: c_{-r, k^{\prime}+\frac{\phi}{\phi_{o}} \frac{2 \pi}{L}}^{\dagger} c_{-r, k^{\prime}+\frac{\phi}{\phi_{o}} \frac{2 \pi}{L}}: \\
& +\frac{g_{4}}{2 L} \sum_{r, k, k^{\prime}}: c_{r, k+\frac{\phi}{\phi_{o}} \frac{2 \pi}{L}}^{\dagger} c_{r, k+\frac{\phi}{\phi_{0}} \frac{2 \pi}{L}}:: c_{r, k^{\prime}+\frac{\phi}{\phi_{O}} \frac{2 \pi}{L}}^{\dagger} c_{r, k^{\prime}+\frac{\phi}{\phi_{o}} \frac{2 \pi}{L}}:
\end{aligned}
$$

where the normal ordering subtracts the infinite ground state density of type-r fermions. We observe that $N_{r} \equiv$ $\sum_{r, k}: c_{r, k+\frac{\phi}{\phi_{o}} \frac{2 \pi}{L}}^{\dagger} c_{r, k+\frac{\phi}{\phi_{O}} \frac{2 \pi}{L}}:$ is an integer number of the excited fermions for any value of $\phi$. Also, $N_{r}$ is a good quantum number because $\left[H, N_{r}\right]=0$ and does not change continuously as implied in the continuum approximation. Here, we note that the interaction parameter $g_{2}\left(g_{4}\right)$ is introduced to describe the forward scattering between different(same) branch particles excited from the fermi level. In order to perform the thermal average of the PC, we consider the energy levels of the excited states. Because the excitation energy in the interaction part is completely determined by the charge excitation number, $N$, and the current excitation number, $J$, the parity effect does not appear. Therefore, we obtain for the zero mode of the interaction part,

$$
H_{i n t}^{o}=\frac{1}{2 L}\left(\frac{g_{4}+g_{2}}{2}\right) N^{2}+\frac{1}{2 L}\left(\frac{g_{4}-g_{2}}{2}\right) J^{2} .
$$

Here, flux $\phi$ does not affect the interaction Hamiltonian. The PC is known to be determined by the current excitation number $J$ and the AB flux $\phi$. Since the charge 
excitation degree is decoupled from the current part, it is sufficient to consider the total current excitation contribution only.

$$
H_{J}^{o}=\frac{v_{F} \pi}{2 L}\left(\left(J+k_{J}\right)^{2}+2\left(J+k_{J}\right) \frac{2 \phi}{\phi_{o}}\right)+\frac{g}{2 L} J^{2},
$$

where $g=\frac{g_{4}-g_{2}}{2}$.

In the LL Hamiltonian, the zero modes are decoupled from the non-zero mode. Here, we write down the zero mode of the partition function

$$
Z_{o}=C \sum_{m} e^{-\beta\left[\frac{\pi v_{F}}{2 L}\left(\left(2 m+k_{J}\right)^{2}+2\left(2 m+k_{J}\right) \frac{2 \phi}{\phi_{o}}\right)+\frac{g}{2 L}(2 m)^{2}\right]},
$$

where terms irrelevant in the current calculation are absorbed in constant C. When there is no charge excitation, the eigenvalue of operator, $J$, becomes an even integer, $2 m$. It is necessary to carry out the exact summation process instead of the integration process of the continuum approximation to incorporate the $\mathrm{AB}$ flux effect and the parity effect correctly. The result is given by

$$
Z_{0}=C \theta_{3}\left(\frac{i \pi v_{F} \beta}{L}\left(k_{J}+\frac{2 \phi}{\phi_{o}}\right), e^{-\frac{2 \pi \eta v \beta}{L}}\right) e^{-\frac{2 \pi v_{F} \beta}{L} k_{J} \frac{\phi}{\phi_{o}}},
$$

where $\mathrm{C}$ is a constant which does not depend on the flux $\phi$ and $\theta_{3}(v, q)=\sum_{n=-\infty}^{\infty} q^{n^{2}} e^{i 2 n v}$ is the Jacobi theta function.

The current can be obtained as before calculating the total momentum, $-\frac{e}{L} \sum_{n=-N_{o-}-N_{-}}^{N_{o+}+N_{+}} \frac{2 \pi}{L}\left(n+\frac{\phi}{\phi_{o}}\right)$, and represented by the excitation numbers,

$$
I=-\frac{e v_{F}}{L}\left(J+k_{J}+\frac{2 \phi}{\phi_{o}}\right)
$$

where $v_{F}=\frac{N_{o}}{2} \frac{2 \pi}{L}$. Here we note that $I_{o} \equiv \frac{e v_{F}}{L}=\frac{2 \pi v_{F}}{L \phi_{o}}$ in unit $\hbar=1$. With Eq. (10) and Eq. (12), we readily obtain the expectation value of $\mathrm{PC}$ given by

$$
\langle I\rangle=-\frac{\partial}{\partial \phi}\left[-\frac{1}{\beta} \ln Z_{o}\right]-I_{o} \frac{2 \phi}{\phi_{o}},
$$

which has a final form

$$
\langle I\rangle=-I_{o}\left[k_{J}+\frac{2 \phi}{\phi_{o}}-2 \sum_{n=1}^{\infty}(-1)^{n} \frac{\sinh \left(k_{J}+\frac{2 \phi}{\phi_{o}}\right) \frac{n T^{*}}{T}}{\sinh \frac{\eta v}{v_{F}} \frac{n T^{*}}{T}}\right],
$$

where $T^{*} \equiv \frac{2 \pi v_{F}}{k_{B} L}$ is the characteristic temperature. Here, we used

$$
\frac{\partial_{\alpha} \theta_{3}(i \alpha, q)}{\theta_{3}(i \alpha, q)}=4 \pi i \sum_{n=1}^{\infty}(-1)^{n} \frac{q^{n}}{1-q^{2 n}} \sinh 2 \pi n \alpha,
$$

which is valid when $|\alpha| \leq-\frac{1}{2 \pi} \ln q[11]$.
When the interaction preserves the left-right symmetry, $g_{2}=g_{4}$ i.e. $g=0$ [国, we observe that there is no contribution from the interaction at all temperatures. This relation, $g=0$, is not satisfied generally. Chiral edge currents in a quantum Hall annulus gives an example[12]. Symmetry breaking due to an AB flux penetrating annulus induces the PCs. The inner and the outer edge currents consist the left and the right branch respectively in a conventional LL. The interaction strength between the edges can be represented by the $g_{2}$ parameter. Because the interedge interaction is weaker than the intraedge one, we observe that $g>0$.

In order to determine the $\mathrm{PC}$ when $g \neq 0$, it is necessary to determine the possible values of $k_{J}$. Using Eq. (11) and the valid range of Eq. (15) with $\alpha=\frac{\pi v_{F} \beta}{L}\left(k_{J}+\frac{2 \phi}{\phi_{o}}\right)$ and $q=\exp \left(-\frac{2 \pi \eta v \beta}{L}\right)$, we find $k_{J}=0$ or -1 for $0<\phi / \phi_{o}<1 / 2$ and $k_{J}=0$ or 1 for $-1 / 2<\phi / \phi_{o}<0$. The constraint on the current number further reduces to $k_{J}=0$ for the odd parity and $k_{J}=-1(+1)$ for positive(negative) $\phi$ for the even parity. We see that this is consistent with the values of $k_{J}$ which we obtained from the physical argument previously.

We examine the zero temperature limit of the PC for positive $g$ using Eq. (14) and obtain results which are same to those in non-interacting case [8]:

$$
\begin{array}{ll}
\langle I\rangle=-\frac{4 \pi v_{F} \phi}{L \phi_{o}^{2}}, & \text { odd parity, } \\
\langle I\rangle=\frac{2 \pi v_{F}}{L \phi_{o}}\left(1-\frac{2|\phi|}{\phi_{o}}\right) \operatorname{sgn} \phi, & \text { even parity. }
\end{array}
$$

The above results clearly show that the $\mathrm{PC}$ is not renormalized by interaction at zero temperature contrary to the results of previous calculations [6. This result is consistent with microscopic derivations 13].

For finite temperatures, the PC shows a qualitatively different behavior from the non-interacting case as shown in Fig. 1. We, first, consider the even parity case. It is sufficient to consider the positive $\phi$ regime $\left(k_{J}=-1\right)$. We can see from the partition function in Eq. (10) that, in the non-interacting case at the ground state, $m=0$. The first excited state, $m=+1$, has a lower energy than that of the state, $m=-1$. This reduces the value of current because value of the current has the opposite sign of the momentum as shown in Eq. (12). The interacting term, however, does not favor any particular current direction because of the equal energy cost for $m= \pm 1$. Therefore, inclusion of the interaction term makes the current reduction smaller and, thus, the amplitude is enhanced compared to the non-interacting case(Fig. 2). For the odd parity, the PC is just the translation of the even parity case by $\phi_{o} / 2$ as can be seen in Eq. (14) with $k_{J}=0$. The reduction of amplitude is also smaller than that of the non-interacting case. We note that the translational symmetry between the odd and the even parity case and the magnetization direction due to the $\mathrm{PC}$ are consistent 


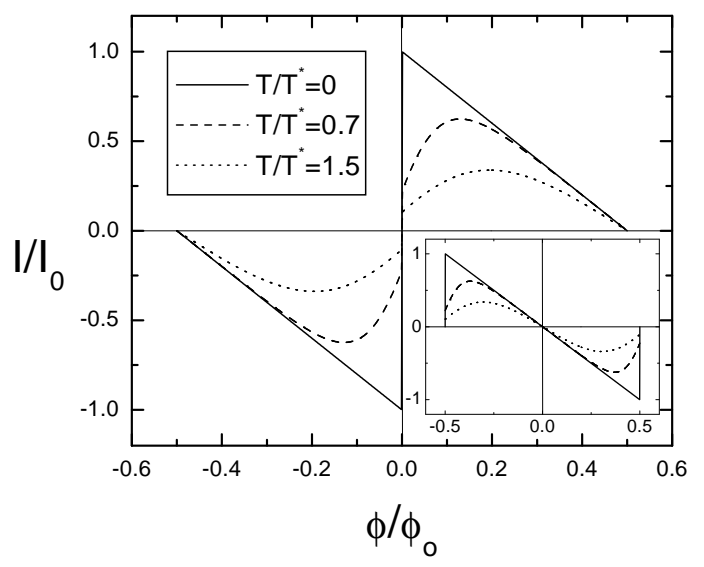

FIG. 1: PCs at several temperatures for the even parity, when $g=0.05$. The figure in the inset shows the odd parity case.

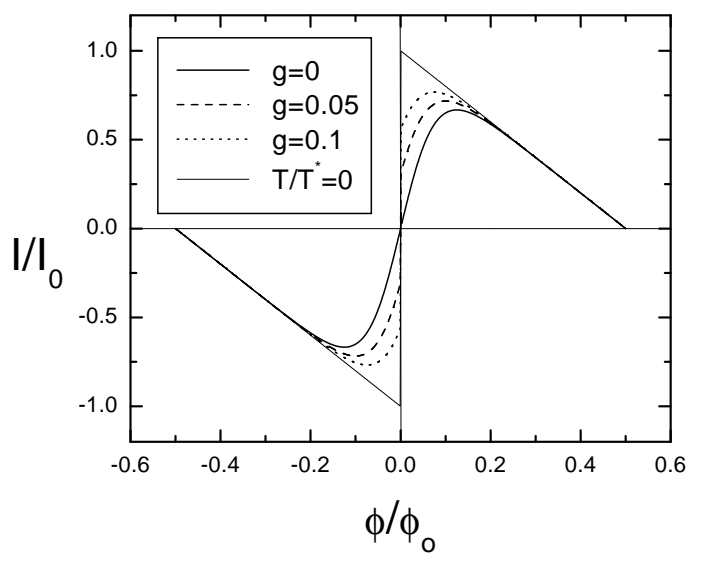

FIG. 2: PCs for several interaction parameter $g$ values at $T / T^{*}=0.5$. The amplitudes of PCs are enhanced as $\mathrm{g}$ increases. The zero temperature current for an arbitrary $g$ is a guide for eye.

with the Legget's theorem 14.

Several groups have carried out numerical calculations for PCs in interacting systems with disorder 15, 16, 17. In those cases interactions preserve the left-right symmetry and, thus, they correspond to the case, $g=0$ in LL scheme. Lanczos method [15] showed that, for a clean ring, the amplitude of $\mathrm{PC}$ is independent of the interaction strength away from half-filling. In the presence of disorder, however, the amplitude become dependent on the interaction strength. Lanczos method for a dirty ring 15] and Monte Carlo simulation for a electron interacting with a diffuse environment[16] demonstrated that the mplitude is supressed, while it is enhanced in DMRG calculation for a disordered ring 17].

In summary, we have developed a LL theory in a meso- scopic ring. In mesoscopic systems like AB ring, the level discreteness plays an important role. We obtained the zero mode in the LL Hamiltonian by exact summation instead of continuum integration. At zero temperature, the PC generally does not depend on the interaction, which is consistent with microscopic derivations. At finite temperatures, amplitude of the $\mathrm{PC}$ is enhanced with respect to the non-interacting case and its behavior confirms the Legget's theorem.

This work was partly supported by the Korea Research Foundation(99-005-D00011) and the Korea Science and Engineering Foundation through Center for Strongly Correlated Materials Research(SNU).

[1] F. D. M. Haldane, J. Phys. C 14, 2585(1981).

[2] J. Voit, Rep. Prog. Phys. 58, 977(1995).

[3] A. O. Gogolin, A. A. Nersesyan, and A. M. Tsvelik, Bosonization and Strongly Correlated Systems (Cambridge University Press, Cambridge, 1998).

[4] J. von Delft and H. Schoeller, Ann. Phys.(Leipzig) 7, 225(1998)

[5] H. J. Schulz, G. Guniberti, and P. Pieri, in Field Theories for Low-Dimensional Condensed Matter Systems, edited by G. Morandi et al.(Springer, 2000).

[6] D. Loss, Phys. Rev. Lett. 69, 343(1992); D. Schmeltzer, Phys. Rev. B 47, 7591(1993);D. Schmeltzer and R. Berkovits, Phys. Lett. A 253, 341(1999);D. Schmeltzer, Phys. Rev. B 63, 125332(2001).

[7] N. Byers and C. N. Yang, Phys. Rev. Lett. 7, 46(1961).

[8] H.-F. Cheung, Y. Gefen, E. K. Riedel and W.-H. Shih, Phys. Rev. B. 37, 6050(1988); D. Loss and Goldbart, ibid. 43, 13762(1991).

[9] L. P. Lévy, G. Dolan, J. Dunsmuir, and H. Bouchiat, Phys. Rev. Lett. 64, 2074(1990); V. Chandrasekhar, R. A. Webb, M. J. Brady, M. B. Ketchen, W. J. Gallagher, and A. Kleinsasser, ibid. 67, 3578(1991); D. Mailly, C. Chapelier, and A. Benoit, ibid. 70, 2020(1993).

[10] See, for instance, H. Fukuyama, in Mesoscopic Physics and Electronics, edited by T. Ando et al.(Springer, Berlin, 1998).

[11] H. Bateman and A. Erderly, Higher Transcendental Functions(McGraw Hill, 1954).

[12] D. J. Thouless and Y. Gefen, Phys. Rev. Lett. 66, 806(1991); Y. Gefen and D. J. Thouless, Phys. Rev. B 47, 10423(1993); S. Kettemann, ibid. 55, 2512(1997).

[13] A. Müller-Groeling, H. A. Weidenmüller and C. H. Lewenkopf, Europhys. Lett. 22, 193(1993); A.MüllerGroeling and H. A. Weidenmüller, Phys. Rev. B. 49, 4752(1994).

[14] A. J. Legget, in Granular Nanoelectronics, edited by D. K. Ferry, J. R. Barker, and C. Jacoboni, NATO ASI Ser. B 251, 279(Plenum, New York, 1991).

[15] G. Bouzerar, D. Poilblanc, and G. Montambaux, Phys. Rev. B 49, 8258(1994).

[16] D. S. Golubev, C. P. Herrero, and A. D. Zaikin, condmat/0205549.

[17] P. Schmitteckert, R. A. Jalabert, D. Weinmann, and J.L. Pichard, Phys. Rev. Lett. 81, 2308(1998). 